\title{
Ionization Mechanism in Surface Plasmon Enhanced Laser Desorption/Ionization*
}

\author{
Keishiro Nagoshi ${ }^{\dagger}$ Kazuhiro Sakata, Kohei Shibamoto, and Takashi Korenaga \\ Department of Chemistry, Tokyo Metropolitan University, \\ 1-1 Minami-Osawa, Hachioji, 192-0397 Tokyo, Japan \\ (Received 17 November 2008; Accepted 9 January 2009; Published 14 February 2009)
}

\begin{abstract}
We researched contribution of a charge transfer (CT) effect to desorption/ionization mechanism in our ultra-high sensitive laser desorption/ionization mass spectrometry based on surface plasmon (SP) excitation (SPLDI-MS). A quantity of CT electrons between a metal surface and sample molecules estimated from Raman measurement is correlated to a mass signal intensity of sample molecule in our SPLDI-MS method. A sample system with a larger quantity of CT electrons gave a higher mass signal intensity. Efficient use of the CT effect would lead to development of a higher sensitive SPLDI-MS and this development would contribute to advancement of various fields.
\end{abstract}

[DOI: $10.1380 /$ ejssnt.2009.93]

Keywords: Gold nanoparticles; Laser desorption/ionization; Surface plasmon; Surface-enhanced Raman scattering; Charge transfer effect

\section{INTRODUCTION}

Surfaces of nanoparticles and nanostructures have unique properties that are different from their bulk phase because these materials have huge specific surface area and nano-sized effects. Surface plasmon (SP) excitation, which is one of these unique physicochemical properties, is well known for high optical responsivity. Therefore, the SP excitation has attracted much attention and has been widely applied for many fields such as sensing techniques [1], nano fabrications [2], and ultra-high sensitive spectroscopy [3].

Matrix-assisted laser desorption/ionization mass spectrometry (MALDI-MS) method has been widely used for an analysis of biomolecules, proteins and synthetic polymers $[4,5]$. However, the MALDI-MS, which is a very useful analytical method, has mainly three problems caused by an addition of excess matrix molecules to sample molecules. First problem is that an analytical performance of the MALDI-MS method strongly depends on a combination of matrix molecules with sample molecules and absorbance of matrix molecules at wavelength of irradiating laser. Second one is an inhibition of back ground signals from matrix molecules in a low-mass range. Third one is a lack of reproducibility due to inhomogeneous cocrystallization of matrix molecules with sample molecules.

In order to solve these matrix problems, many researchers have developed surface-assisted LDI-MS (SALDI-MS) methods as a matrix-free LDI-MS method. For example, ionization substrates of these SALDI-MS methods such as porous silicon, pyroelectric ceramics and $\mathrm{TiO}_{2}$ sol-gel film have been proposed [6-8]. Although these matrix problems are removed by appearance of SALDI-MS methods, these SALDI-MS methods are not widely used because of too lower sensitivity than the MALDI-MS method in practical use. Their low sensitivity may be caused by an assumption that desorp-

\footnotetext{
* This paper was presented at International Symposium on Surface Science and Nanotechnology (ISSS-5), Waseda University, Japan, 9-13 November, 2008.

†Corresponding author: nagoshi-keishiro@tmu.ac.jp
}

tion/ionization mechanisms in the SALDI-MS methods are similar to desorption/ionization mechanism based on rapid thermal energy supply in the MALDI-MS method. In fact, it is difficult to explain these results in the SALDIMS methods from a viewpoint of only rapid thermal energy supply. Because desorption/ionization process is one of surface reactions, and there should be other effects on the surface. So their discussions may lack a contribution of surface effect. Therefore, potentially existing surface effects (not thermal energy supply) should be utilized as energy supply in the SALDI-MS methods. Thus, we focused the SP excitation, which has huge surface-enhanced effects, and then we succeeded to develop a novel SALDIMS (SPLDI-MS) method with ultra-high sensitivity using gold nanoparticles (AuNPs) [9]. However, the energy supply mechanisms based on the SP excitation in the SPLDIMS method are not clarified in detail.

The SP excitation generates a huge density of excited electrons on the metal surface. It is known that the huge density of excited electrons induces two closely-associated surface effects. One is an electromagnetic (EM) effect which is resulting in the local enhancement of the electromagnetic field. Another one is a charge transfer (CT) effect which is closely related to a property of adsorbed molecules. One of above-mentioned SP applications is surface-enhanced Raman scattering (SERS) [10]. The SERS is a spectroscopic technique which remarkably enhances Raman signals $[11,12]$, and its enhancement mechanism is explained by the CT effect $[13,14]$ and the EM effect [15]. Therefore, these two effects should be discussed in our SPLDI-MS method. Since the CT effect would act as direct energy supply from the metal surface to individual adsorbed molecules, we assume that the CT effect is directly related to the ultra-high sensitivity in our SPLDI-MS method.

In this paper, we researched contribution of the CT effect based on the SP excitation to a desorption/ionization mechanism in our SPLDI-MS method. Understanding of the contribution of the CT effect leads to development of a super SALDI-MS method which enables to detect single-molecule. We expect that such SALDI-MS method is widely applied in many studies such as biochemistry, environmental chemistry, imaging techniques, and so on. 


\section{EXPERIMENTS}

\section{A. Materials}

AuNPs $\left(60 \mathrm{~nm}, 2.6 \times 10^{10}\right.$ particles $\left./ \mathrm{ml}\right)$ with its narrow distribution in size were purchased from BBInternational. No surfactant or capping agent was used for stabilizing the AuNPs. Silicon wafers (N-type, low, (100)) were purchased from Nilaco (Tokyo, Japan). Aminopropyltriethoxy silane (APS) were purchased from ShinEtsu Chemical Co., Ltd. (Tokyo, Japan). Crystal violet (CV) $\mathrm{C}_{25} \mathrm{H}_{30} \mathrm{ClN}_{3}$ molecules were purchased from Sigma-Aldrich, Inc. Rhodamine B (RB) $\mathrm{C}_{28} \mathrm{H}_{31} \mathrm{ClN}_{2} \mathrm{O}_{3}$ molecules were purchased from Kanto Chemical Co., Inc. Malachite green (MG) $\mathrm{C}_{25} \mathrm{H}_{30} \mathrm{ClN}_{3}$ molecules were purchased from Wako Pure Chemical Industries, Ltd.

\section{B. AuNPs substrates Preparation}

Silicon wafers were cut into square substrates $(5 \times 5$ $\mathrm{mm})$. These substrates were heated for $2 \mathrm{~min}$ at $1000^{\circ} \mathrm{C}$ in an oven. This step produced a thermally-oxidized film $\left(\mathrm{SiO}_{2}\right)$ at the $\mathrm{Si}$ surface. These $\mathrm{SiO}_{2} / \mathrm{Si}$ substrates were washed ultrasonically in ethanol, acetone and pure water (2 times for $10 \mathrm{~min}$ ). APS molecules were vapor-deposited onto these $\mathrm{SiO}_{2} / \mathrm{Si}$ substrates under reduced pressure for $5 \mathrm{~h}$. The APS molecules were selected for surface modification because an APS-modified surface attracts AuNPs electrostatically. After washing the substrate ultrasonically to remove excess APS molecules, these substrates were heated for $3 \mathrm{~h}$ at $120^{\circ} \mathrm{C}$ in an oven for binding APS to $\mathrm{SiO}_{2}$ chemically. After washing these substrates ultrasonically again, these substrates were dried in $\mathrm{N}_{2}$. These $\mathrm{APS} / \mathrm{SiO}_{2} / \mathrm{Si}$ substrates were dipped in $10 \%$ AuNPs aqueous solution for $3 \mathrm{~h}$ at $4^{\circ} \mathrm{C}$. AuNPs were immobilized onto these APS $/ \mathrm{SiO}_{2} / \mathrm{Si}$ substrates electrostatically. Finally these substrates (AuNPs substrates) were rinsed with pure water. Surface observation of these AuNPs substrates were examined by scanning electron microscope (SEM ; KEYENCE VK-9700).

\section{Sample molecules preparation}

$\mathrm{CV}, \mathrm{RB}$ and MG molecules as sample molecules were dissolved in pure water at a concentration of $24 \mu \mathrm{M}$. These molecules were adsorbed onto the surface of AuNPs under a saturation condition by dipping the AuNPs substrates into the aqueous solution of the molecules for $30 \mathrm{~min}$, and the substrates were washed with pure water to remove impurities and non-adsorbed molecules.

\section{Instruments}

We used same samples for both Raman measurement and MS measurement. The Raman measurement was carried out by a micro-Raman measurement system (HORIBA HR800) in $300 \mathrm{~s}$ at 488-nm CW excitation (Continuous-Wave Ar laser, $10 \mathrm{~mW}$ ). The cumulated number of the measurement is 2 times. The SPLDI-MS

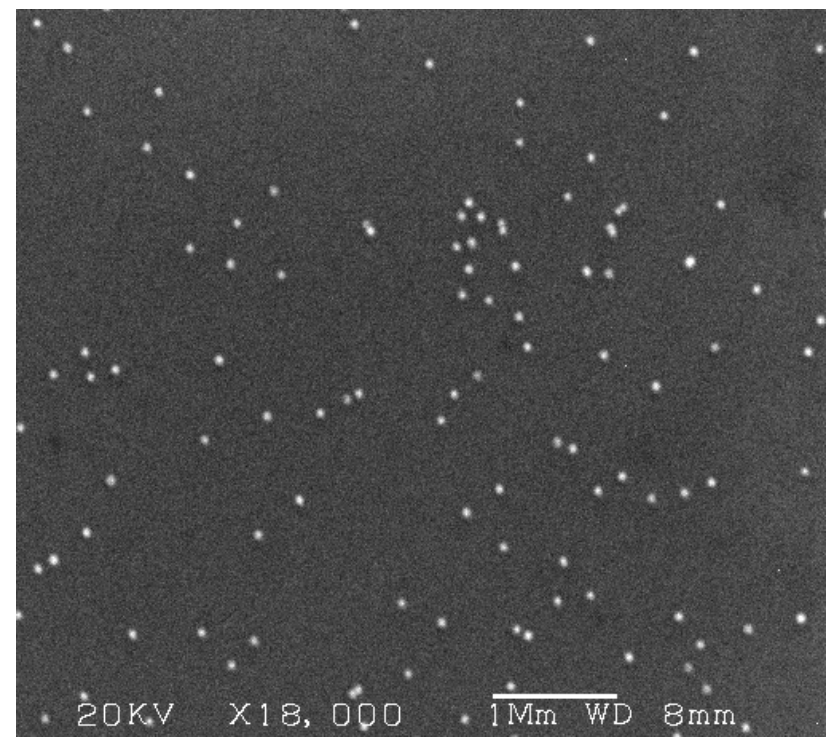

FIG. 1: SEM image of AuNPs substrate.

measurement was carried out by our laboratory-made LDI time-of-flight mass spectrometer at 532-nm pulsed excitation (SHG Nd/YAG laser, $6 \mathrm{~ns}, 10 \mathrm{~Hz}$ ). The 532-nm laser excites an SP band of the AuNP efficiently. These pulses were focused with a $300-\mathrm{mm}$ spherical plano-convex lens of fused silica and were obliquely irradiated onto the AuNPs substrate at the position of $270 \mathrm{~mm}$ from the lens. In our system, the irradiating angle was $45^{\circ}$ and the actual energy fluence on the AuNPs substrate can be estimated about $5.4 \mathrm{~mJ} / \mathrm{cm}^{2}$. The absorption spectrum was carried out by a UV-Vis spectrophotometers (SHIMADZU UV-1600).

\section{RESULTS AND DISCUSSION}

A SEM image of the AuNPs substrate is shown in Fig. 1. A number density of AuNPs on the substrate was approx. 3 particles $/ \mu \mathrm{m}^{2}$. We assumed that the number densities per unit area were almost equal for every sample and were estimated at $1.3 \times 10^{14}$ molecules $/ \mathrm{cm}^{2}$ because $\mathrm{CV}, \mathrm{RB}$ and MG molecules have a similar structure with a carbon in the center and three benzene rings at each apex of a triangle. From this reason, we estimated that the adsorbed molecules of approx. $10 \mathrm{fmol}$ were included within laser spot area $\left(0.13 \mathrm{~mm}^{2}\right)$.

Raman spectra of $\mathrm{CV}, \mathrm{RB}$ and $\mathrm{MG}$ molecules onto AuNPs substrates are shown in Fig. 2. Their Raman signal intensity decreased in order of CV, RB and MG. On the other hand, Raman signals of all samples without addition of AuNPs were not observed in our measurement range (data not shown). This means that Raman signal intensity caused by resonant Raman effect was below detection limit in our measurement system and therefore contribution of resonant Raman effect can be excluded. Thus, it is considered that the Raman signal intensity reflects only a SERS enhancement factor for each molecule. In other words, the effect of SP excitation can be estimated from the Raman signal intensity. It is difficult to 


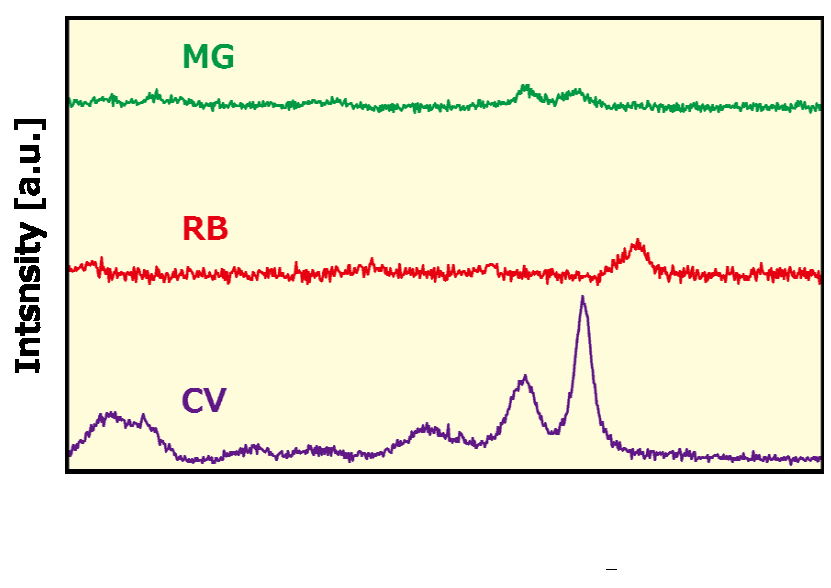

FIG. 2: Raman spectra of CV, RB and MG.

discuss the Raman signal intensity for different molecules; however, in order to estimate the enhancement factor approximately, the Raman signal intensities of aromatic ring C-C stretching band around $1620 \mathrm{~cm}^{-1}$ were compared, and the ratio was $\mathrm{CV} / \mathrm{AuNP}: \mathrm{RB} / \mathrm{AuNP}$ : MG/AuNP $=12: 5: 1$. The reason for a choice of the band is that the C-C stretching band is common among all sample molecules in our experiment and is not shifted by adsorption. On the other hand, co-author, Shibamoto, had reported that the SERS enhancement factor strongly depends on the number density of CT electrons and the density decreased in order of $\mathrm{CV} / \mathrm{AuNP}, \mathrm{RB} / \mathrm{AuNP}$ and $\mathrm{MG} / \mathrm{AuNP}$ [13]. Generally, it is known that the SERS enhancement factor is proportional to the square of the electromagnetic intensity, which is the origin of the EM effect, and the square of the Raman tensor, which is the origin of the CT effect. According to ref. 13, the SERS enhancement factor was roughly proportional to the number density of CT electrons (= CT effect). This means that the EM effect of each sample (CV/AuNP, RB/AuNP and $\mathrm{MG} / \mathrm{AuNP}$ ) contributes to SERS enhancement factor equally in his experiment. Thus, it is considered that the result of Raman measurement in our system reflects a contribution of the CT effect. For this reason, we estimated roughly that the number density of $\mathrm{CT}$ electrons was $\mathrm{CV} / \mathrm{AuNP}: \mathrm{RB} / \mathrm{AuNP}: \mathrm{MG} / \mathrm{AuNP}=12: 5: 1$.

Mass spectra of CV, RB and MG molecules onto AuNPs substrates are shown in Fig. 3. The mass signal intensity of molecular ions $\left(\mathrm{CV}^{+}: \mathrm{m} / z=372, \mathrm{RB}^{+}: \mathrm{m} / z=472\right.$, $\mathrm{MG}^{+}: m / z=329$ ) also decreased in order of $\mathrm{CV}, \mathrm{RB}$ and MG. On the other hand, mass signals of all samples without addition of AuNPs were not observed in our measurement range (data not shown). This means that amount of sample molecular-related ions caused by singleor multi-photon ionization process was below a detection limit in our measurement system and therefore contribution of the single- or multi-photon ionization process can be excluded. Moreover, all these mass spectra with very few fragmentations peaks suggest that we do not need to consider the single- or multi-photon ionization process which induces dissociation of sample molecules. Thus, it is considered that this huge increase of amount of sample molecular ions is induced by only the effect of SP excita-

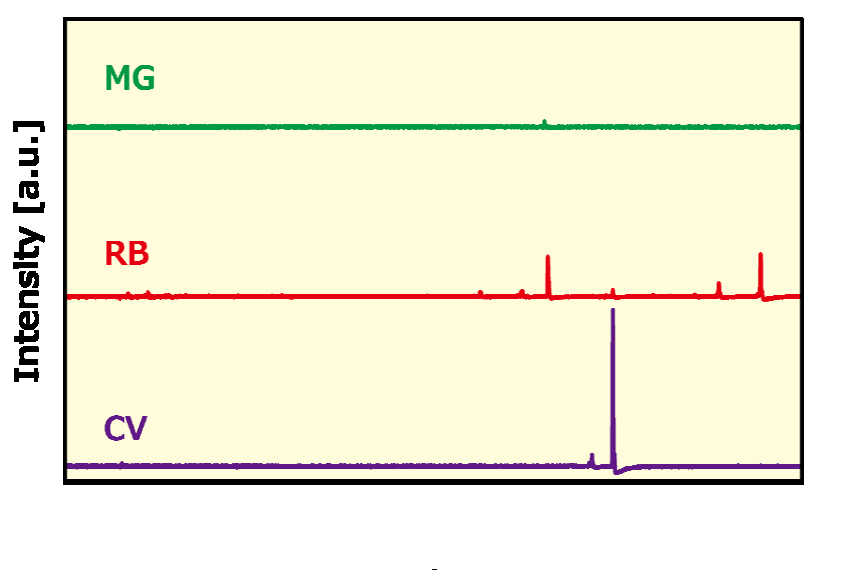

FIG. 3: SPLDI-MS spectra of CV, RB and MG.

tion.

Although the estimation of the enhancement factor from the MS measurement has a similar problem to that from the Raman measurement, the mass signal intensities of molecular ions were compared, and the ratio was $\mathrm{CV}$ : $\mathrm{RB}: \mathrm{MG}=28: 7: 1$. Since a tendency of $\mathrm{CV}>\mathrm{RB}>\mathrm{MG}$ was shown in both measurements, we guessed that a larger number density of CT electrons might give a stronger mass signal intensity. However, in a ratio of signal intensity, the result of the SPLDI-MS measurement was in poor agreement with that of the Raman measurement. We assumed that this poor agreement is related to other influences, which are the following mainly three factors: (i) contribution of the EM effect, (ii) desorption energy of each sample molecule, (iii) difference of laser condition between the SPLDI-MS and the Raman measurement.

Firstly, we discussed the factor (i). The EM effect is a well-known phenomenon that electromagnetic field of an incident light is enhanced remarkably and then localized enhanced electromagnetic field is generated on AuNP surface $[16,17]$. Based on this theory of the EM effect, we should consider the contribution of the EM effect to desorption/ionization mechanism in our SPLDI-MS method [9]. Generally, in LDI-MS method, mass signal intensity is reflected by optical absorption coefficient of sample molecule at an excitation wavelength. In viewpoint of the EM effect, our SPLDI-MS method is regarded as conventional LDI-MS method with high fluence laser irradiation. UV-Vis absorption spectra of $\mathrm{CV}$ and $\mathrm{RB}$ molecules in aqueous solution are shown in Fig. 4. There is a slight difference about optical absorption coefficient at the excitation wavelength (532 $\mathrm{nm}$ ) between $\mathrm{CV}$ and RB molecules in aqueous solution. If the EM effect is a dominant enhancement factor, this difference should reflect that of mass signal intensity. However, our result is against this assumption. Therefore, it is considered that the EM effect is a minor enhancement factor in our sample system.

Secondly, we discussed the factor (ii) which is typically influence in an MS measurement. The result of the MS measurements includes information of desorption energy of each sample, although that of the Raman measurement does not. It is known that desorption energy of $\mathrm{CV}$ molecules is stronger than that of MG molecules. This 


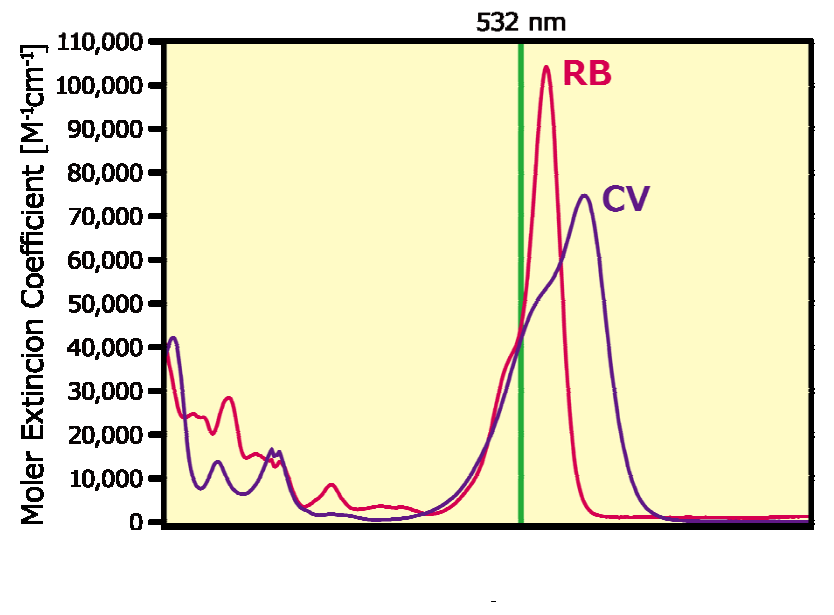

FIG. 4: UV-Vis absorption spectra of CV and RB molecules in aqueous solution.

means that the ratio of $\mathrm{CV} / \mathrm{MG}$ in the Raman measurement should be larger than that in the MS measurement. However, in our results, the ratios in the Raman and the MS measurements were 12 and 28, respectively. Thus, desorption energy of sample molecule is not a dominant factor in our SPLDI-MS measurement.

Finally, we discussed the factor (iii). We used CW laser in the Raman measurement, and pulsed laser in the MS measurement. At present, it is very difficult to compare the CT effect estimated from the Raman measurement, which gives static information, with that from the MS measurement, which gives dynamic information. Even if the $\mathrm{CT}$ effect is a dominant enhancement factor in both measurements, the ratios of signal intensity in both measurements are not exactly equivalent.

From above-mentioned discussions, it is considered that the poor agreement is valid, but these factors do not largely contribute to a tendency of the mass signal intensity. Thus, it is suggested that the CT effect is a dominant enhancement factor in the SPLDI-MS method.

\section{CONCLUSION}

We discussed the contribution of the CT effect in desorption/ionization mechanism in our SPLDI-MS method. We showed that the CT effect is closely related to desorption/ionization mechanism in our SPLDI-MS method. Moreover, a larger CT effect (= larger number of CT electrons) gave a higher mass signal intensity. These results indicate that a direct charge-interaction based on the CT effect enhances desorption/ionization process in our SPLDI-MS method.
[1] K. Kim, H. B. Lee, H. K. Park, and K. S. Shin, J. Colloid Interface Sci. 318, 195 (2008).

[2] D. B. Shao and S. C. Chen, Appl. Phys. Lett. 86, 253107 (2005).

[3] K. Kneipp, Y. Wang, H. Kneipp, L. T. Perelman, and I. Itzkan, Phys. Rev. Lett. 78, 1667 (1997).

[4] M. Karas, D. Bachmann, and F. Hillenkamp, Anal. Chem. 57, 2935 (1985).

[5] K. Tanaka, H. Waki, Y. Ido, S. Akita, Y. Yoshida, and T. Yoshida, Rapid Commun. Mass Spectrom. 2, 151 (1988).

[6] J. Wei, J. M. Buriak, and G. Siuzdak, Nature 399, 243 (1999).

[7] H. Sato, T. Seino, A. Yamamoto, M. Torimura, and H. Tao, Chem. Lett. 34, 1178 (2005).

[8] C.-T. Chen and Y.-C. Chen, Rapid Commun. Mass Spectrom. 18, 1956 (2004).

[9] K. Shibamoto, K. Sakata, and T. Korenaga, J. Phys.
Chem. C (2009), submitted.

[10] M. Fleischman, P. J. Hendra, and A. J. McQuillan, Chem. Phys. Lett. 26, 163 (1974).

[11] D. L. Jeanmaire and R. P. Van Duyne, J. Electroanal. Chem. 84, 1 (1977).

[12] S. Nie and S. R. Emory, Science 275, 1102 (1997).

[13] K. Shibamoto, K. Katayama, and T. Sawada, Chem. Phys. Lett. 433, 385 (2007).

[14] K. Shibamoto, K. Katayama, M. Fujinami, and T. Sawada, Rev. Sci. Instrum. 74, 910 (2003).

[15] G. C. Schatz and R. P. Van Duyne, in: Handbook of Vibrational Spectroscopy, Vol. 1, Eds. J. M. Chalmers and P. R. Griffiths (Wiley, New York, 2002) p. 759.

[16] M. Moskovits, J. Chem. Phys. 69, 4159 (1978).

[17] S. L. McCall, P. M. Platzman, and P. A. Wolff, Phys. Lett. A 77, 381 (1980). 